\title{
Differential Scanning Calorimetric Analysis for Monitoring the Oxidation of Heated Oils*
}

YB Che Man, CP Tan, J Irwandi, JL Liu, S Jinap, HM Ghazall, MSA Yusoff

Faculty of Food Science and Biotechnology

Universiti Putra Malaysia

43H(x) UPM, Serdang, Selangor

Malaysia

E.mail of Corresponding Author: yaakub \& fsb.upm.edu.my

Key words: chemical characteristics, DSC, heated oils, monitoring oxidation.

\section{Introduction}

Lipid oxidation is a major deteriorative reaction affecting edible oils and is a primary concem to manufacturers and consumers from a quality standpoint. Many of the degradation products of edible oils are harmful to human health as they destroy vitamins, inhibit enzymes, potentially cause mutations or cause gastrointestinal irritations. Hence arises the necessity of quality assessment over heated or frying oils, being a special importance in the food industry. There are numerous analytical methods for estimating detcrioration of edible oils. However, these methods are mostly time- and work-consuming methods and require the use of toxic chemicals that are hazardous to the analysts as well as to the environment. Therefore, a rapid and instrumental method is required to monitor and determine the extent of oxidation process in heated oils. Differential scanning calorimetry (DSC) techniques have the potential to be used as a nonchemical method to determine oil quality parameters. The objective of this study was to lay the foundation for the development of DSC Iechnique to investigate the correlation between DSC thermogram parameters and other standard chemical methods in heated oils.

\section{Materials and Methods}

Three different types of edible oils namely, com oil (CO), refined, bleached and decodorised palm olein (RBDPO) and soybean oil (SO) were used in this study. Oil samples $(4.5 \mathrm{~kg})$ were placed in open deep fat fryers (Berto's electrical fryer ALT series. ELT 8B, Montegrotto T., Padova, Italy) and heated at $180^{\circ} \mathrm{C}$. About $200 \mathrm{~g}$ sample were collected in screw-capped amber bottles at the start of the $180^{\circ} \mathrm{C}$ $(0 \mathrm{~h})$ and at $2 \mathrm{~h}$ interval for a period of $12 \mathrm{~h}$. The DSC method was based on the cooling thermogram of oil samples at scanning ratc of $1^{\circ} \mathrm{C} / \mathrm{min}$ from -30 $10-85^{\circ} \mathrm{C}$. Two DSC parameters of this single crystallisation peak namely peak temperature and enthalpy were determined. In addition to DSC method, deterioration of heated oils were also quantified by means of seven chemical methods. These chemical methods were total polar compounds (TPC), iodine value (IV), free fatty acid (FFA), anisidine value (AnV), peroxide value $(\mathrm{PV})$, totox value $(\mathrm{TxV})$ and ratio of $\mathrm{C} 18: 2 / \mathrm{Cl6}$ :0.

\section{Results and Discussion}

Generally, experimental results of the TPC. FFA, AnV, PV and TxV showed an increase with heating time. In contrast, the IV and C18:2/C16:0, DSC peak temperature and enthalpy of heated oils decreased with the ascending time of heating. In this study, heated oils exhibited a simple thermogram after cooling in the DSC with a well-defined single crystallisation peak. The DSC traces are affected in a systematic way by the increased in heating duration. In general, as the time of heating increased, the peak of crystallisation shifted to lower temperatures and enthalpy decreased dramatically. According to Hagemann et al. and Tallent (1972), slow-scan cooling curves of unsaturated triglycerides generally showed an exotherm at approximately the melting point of the $\alpha$ form. Berger et al. and Akchurst (1966) also reported that the typical cooling thermogram of the edible oil shows a dominant exothermic peak at supercooling temperature range. Since the contents of unsaturated fatty acids were higher in $\mathrm{CO}$ and $\mathrm{SO}$ in comparison to RBDPO, the crystallisation peaks in $\mathrm{CO}$ and SO were more apparent than RBDPO. In heated oils, the oxidation products such as polar compounds, dimers, polymers, hydroperoxides and aldehydes increased during heating process. As their level increase, such compounds would contribute to the changes in DSC crystallisation peak parameters. Further study on the crystallisation behaviour of polar and nonpolar fraction of heated oils derived supportive evidence to this phenomenon. The result showed that the crystallisation peak was not observed in the polar fraction of heated oils. This may be attributed to the disappearance of unsaturated triglycerides and the formation of degradation products in polar fraction. The DSC thermogram pa. rameters showed excellent correlations with the standard analytical measurements regardless the oils degradation treatment in this case and independent of the edible oil source. The coefficients of correlation for each comparison were also highly significant $(\mathrm{P}<0.01)$. The peak temperature was strongly negative correlation to TPC. FFA, AnV, PV and TxV, and was highly positive correlation to IV and C18:2/C16:0. In contrast, the peak enthalpy showed significant positive correlation to TPC, FFA, AnV, PV and $T x V$, and was highly significant negative correlation to IV and C18:2/Cl6:0.

\section{Conclusions}

The results of the present study suggest that the DSC method can be a reliable and efficient alternative to existing methods for monitoring the extent of lipid oxidation in various edible oils, with no need for chemical reagents and delicate skills. Our results represent a new technique to be used for the assessment of oils of different quality.

\section{Benefits from the study}

Inaccurate monitoring of oil quality can either risk the public health or cause financial losses to the food industry. Moreover, fast and reliable methods for testing both the immediate quality and the expected oxidation stage of heated oils are in great demand. Therefore. additional efforts are necessary to 
develop potential new techniques. such as DSC analysis, that may be used in food industry.

\section{Literature cited in the text}

Berger, K.G.. and Akehurst, E.E. 1966.

Some application of differential thermal analysis to oils and fats. J. Fd Technol. 1: 237.247.

Hagemunn, J.W. and Tallent, W.H. 1972. Differential scanning calorimetry of single acid triglycerides: Effect of chain length and unsaturation. J. Am. Oil Chem. Soc. 49: 119-123.

\section{Project Publications in} Refereed Journals

Biliaderis, C.G. 1983. Differential scanning calorimetry in $f(x) d$ research - A review. Ford Chem. 10: 234-265.

Clark. W.L. and Serbia, G.W. (') I. Safely aspects of frying fats and oils. Food Techno. 45(2): 84-86, 88, 89. 94.

Paul, S. and Mittal. G.S. 1 $\% \%$. Dynamicn of favoil degradation during frying based on physical propertice. J. Food Proce. Eing. 19: 201-221.

Paul. S. and Mittal, G.S. 1997. Regulating the use of degraded oilfat in decp-fat/vil fendl frying. Crit. Rev. Fionl Sci. Nutr. 37: 6.35. $0 \times 12$

White, P.J. (9)1. Methends for meusuring changes in deep-fat trying wils. Foest Trchuol. +5(2). 75-80).

\section{Project Publications in} Conference Proceedings None.

Graduate Research

None.

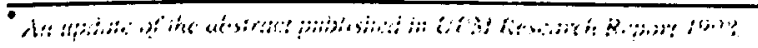

